\title{
Protection from non-alcoholic steatohepatitis and liver tumourigenesis in high fat-fed insulin receptor substrate-1-knockout mice despite insulin resistance
}

\author{
A. Nakamura $\cdot$ K. Tajima $\cdot$ K. Zolzaya $\cdot$ K. Sato • \\ R. Inoue - M. Yoneda $\cdot$ K. Fujita • Y. Nozaki • \\ K. C. Kubota • H. Haga • N. Kubota • Y. Nagashima • \\ A. Nakajima $\cdot$ S. Maeda $\cdot$ T. Kadowaki $\cdot$ Y. Terauchi
}

Received: 28 May 2012 / Accepted: 27 July 2012 /Published online: 7 September 2012

(C) Springer-Verlag 2012

\begin{abstract}
Aims/hypothesis Epidemiological studies have revealed that obesity and diabetes mellitus are independent risk factors for the development of non-alcoholic steatohepatitis (NASH) and hepatocellular carcinoma. However, the debate continues on whether insulin resistance as such is directly associated with NASH and liver tumourigenesis. Here, we investigated the
\end{abstract}

A. Nakamura and K. Tajima contributed equally to this work.

Electronic supplementary material The online version of this article (doi:10.1007/s00125-012-2703-1) contains peer-reviewed but unedited supplementary material, which is available to authorised users.

A. Nakamura $\cdot$ K. Tajima $\cdot$ K. Zolzaya $\cdot$ K. Sato $\cdot$ R. Inoue $\cdot$

Y. Terauchi $(\bowtie)$

Department of Endocrinology and Metabolism,

Graduate School of Medicine, Yokohama City University,

3-9 Fukuura, Kanazawa-ku,

Yokohama 236-0004, Japan

e-mail: terauchi-tky@umin.ac.jp

M. Yoneda $\cdot$ K. Fujita $\cdot$ Y. Nozaki $\cdot$ A. Nakajima $\cdot$ S. Maeda Division of Gastroenterology, Graduate School of Medicine, Yokohama City University,

Yokohama, Japan

K. C. Kubota $\cdot$ H. Haga

Department of Surgical Pathology, Hokkaido University Hospital,

Sapporo, Japan

N. Kubota $\cdot$ T. Kadowaki

Department of Diabetes and Metabolic Diseases,

Graduate School of Medicine, University of Tokyo,

Tokyo, Japan

Y. Nagashima

Department of Molecular Pathology,

Graduate School of Medicine, Yokohama City University,

Yokohama, Japan incidence of NASH and liver tumourigenesis in $\operatorname{Irs} 1^{-/}$mice subjected to a long-term high-fat (HF) diet. Our hypothesis was that hepatic steatosis, rather than insulin resistance may be related to the pathophysiology of these conditions.

Methods Mice (8 weeks old, C57Bl/6J) were given free access to standard chow (SC) or an HF diet. The development of NASH and liver tumourigenesis was evaluated after mice had been on the above-mentioned diets for 60 weeks. Similarly, $\operatorname{Irs} 1^{-/}$mice were also subjected to an HF diet for 60 weeks.

Results Long-term HF diet loading, which causes obesity and insulin resistance, was sufficient to induce NASH and liver tumourigenesis in the $\mathrm{C} 57 \mathrm{Bl} / 6 \mathrm{~J}$ mice. Obesity and insulin resistance were reduced by switching mice from the HF diet to $\mathrm{SC}$, which also protected these mice against the development of NASH and liver tumourigenesis. However, compared with wild-type mice fed the HF diet, Irs $1^{-/-}$mice fed the HF diet were dramatically protected against NASH and liver tumourigenesis despite the presence of severe insulin resistance and marked postprandial hyperglycaemia.

Conclusions/interpretation IRS-1 inhibition might protect against HF diet-induced NASH and liver tumourigenesis, despite the presence of insulin resistance.

Keywords High-fat diet · Insulin receptor substrate-1 . Insulin resistance $\cdot$ Liver tumourigenesis $\cdot$ Non-alcoholic steatohepatitis
Abbreviations
ALT
Alanine aminotransferase
HCC
Hepatocellular carcinoma
$\mathrm{H} \& \mathrm{E}$
Haematoxylin and eosin
$\mathrm{HF}$ High-fat
HOMA-IR HOMA of insulin resistance 
NAFLD Non-alcoholic fatty liver disease

NASH Non-alcoholic steatohepatitis

PI3K Phosphoinositide 3-kinase

SC Standard chow

\section{Introduction}

The prevalence of obesity and diabetes has been increasing globally over the past 30 years $[1,2]$. These diseases not only increase cardiovascular risk, but also cancer risk and mortality rates $[3,4]$. Especially, hepatocellular carcinoma (HCC), the fifth most common cancer and the third leading cause of cancer death worldwide [5], accounts for the largest increase in cancer and mortality risk in individuals with obesity or diabetes $[3,4]$. Certain cases of HCC may be associated with infection with hepatitis $\mathrm{B}$ or $\mathrm{C}$ virus, or chronic alcohol use. However, an increasing number of cases are associated with non-alcoholic fatty liver disease (NAFLD). NAFLD encompasses a clinicopathological spectrum of diseases ranging from isolated hepatic steatosis to non-alcoholic steatohepatitis (NASH), the more aggressive form of fatty liver disease, which may progress to cirrhosis and cirrhosis-related complications including HCC. The prevalence of NAFLD, including NASH, is also increasing in parallel with the growing obesity and diabetes epidemics [5].

Although the causal relationships between obesity or diabetes, and NASH or liver tumourigenesis have not yet been clearly elucidated, it is assumed that the insulin resistance associated with obesity and diabetes is involved in the development of hepatic steatosis and inflammation in the liver, which may progress to NASH and liver tumourigenesis [5]. Indeed, long-term high-fat (HF) diet loading, which can induce obesity and insulin resistance, was sufficient to induce NASH and liver tumourigenesis in $\mathrm{C} 57 \mathrm{Bl} / 6 \mathrm{~J}$ mice [6-8]. Thus, this experimental model supports the above-mentioned concept.

IRS-1 and -2 exhibit a high structural homology, are abundantly produced in the liver and are thought to be responsible for transmitting insulin signalling from the insulin receptor to intracellular effectors in the regulation of glucose and lipid homeostasis $[9,10]$. Insulin receptor signalling can be almost exclusively mediated by IRS-1 and IRS-2 in the liver [10]; indeed, a dominant role of IRS-1 has been observed during nutrient excess [11]. Moreover, HF diet-fed liver-specific Irs $1^{--}$mice displayed severe insulin resistance, but not hepatic steatosis [11]. Also, mice with acyl-CoA:diacylglycerol acyltransferase (DGAT)2 overabundance in the liver reportedly developed hepatic steatosis without abnormal plasma glucose and insulin levels [12], while liver-specific phosphoinositide 3-kinase (PI3K) p110 $\alpha$-knockout $\left(\mathrm{Pik}_{3} \mathrm{Ca}^{-/-}\right)$mice fed an HF diet were protected against hepatic steatosis without ameliorating HF diet-induced glucose intolerance [13]. Mice with genetic defects or targeted overexpression, such as hepatocyte- specific Pten-knockout mice and Pik3ca transgenic mice $[14,15]$, have been reported to be models of NAFLD and liver tumourigenesis, but might not reflect the natural aetiology of NASH and liver tumourigenesis in human participants. Rather, insulin sensitivity was improved in hepatocytespecific Pten-knockout mice, compared with wild-type mice [14], and Pik3ca transgenic mice exhibited better glucose tolerance than wild-type mice [15]. Therefore, liver steatosis can occur independently of insulin resistance.

In the present study, we investigated the effect of a longterm HF diet on the development of NASH and liver tumourigenesis using $\mathrm{C} 57 \mathrm{Bl} / 6 \mathrm{~J}$ male mice. Next, we performed similar experiments in which the HF diet was switched to a standard chow (SC) diet to clarify the effect of improved insulin resistance on the development of these diseases. We also investigated the incidence of NASH and liver tumourigenesis in $I r s 1^{-/-}$mice subjected to a longterm HF diet. The hypothesis behind this part of the study was that hepatic steatosis, rather than insulin resistance, may be related to the pathophysiology of these conditions.

\section{Methods}

Animals Mice $\left(\operatorname{IrS} 1^{-/}\right)$were generated as described elsewhere [16]. We backcrossed these mice with $\mathrm{C} 57 \mathrm{Bl} / 6 \mathrm{~J}$ mice more than nine times. Male littermates derived from the intercrosses were fed an SC diet until 8 weeks of age and then had free access to the SC diet or an HF diet. In the dietary switch experiment, 8 -week-old $\mathrm{C} 57 \mathrm{Bl} / 6 \mathrm{~J}$ male mice were subjected to the HF diet for 30 weeks and then switched to the SC diet for the next 30 weeks; these mice were then compared with mice from the same genetic background that had received the HF diet for the entire 60 weeks. The mice were housed under a $12 \mathrm{~h}$ light-dark cycle. The animals were maintained according to standard animal care procedures based on institutional guidelines. These experiments involving animals were approved by the local Ethics Committee of the Yokohama City University.

Diet protocol We used an SC diet (MF; Oriental Yeast, Tokyo, Japan) and an HF diet (High-Fat Diet 32; Clea Japan, Tokyo, Japan). The composition of each of these diets is shown in electronic supplementary material (ESM) Table 1 . The fatty acid composition of the HF diet consisted of $22 \%(\mathrm{wt} / \mathrm{wt})$ saturated fatty acid (12.6\% palmitic acid, $7.5 \%$ stearic acid) and $77 \%(\mathrm{wt} / \mathrm{wt})$ unsaturated fatty acid (64.3\% oleic acid, $10.2 \%$ linoleic acid).

Measurement of biochemical variables Blood glucose levels were measured using a portable glucose meter and Glutest Neo (Sanwa Chemical, Nagoya, Japan). Insulin levels were determined using an insulin ELISA kit (Morinaga, Yokohama, 
Japan). Plasma alanine aminotransferase (ALT) levels were assayed using an enzymatic method (Wako Pure Chemical, Osaka, Japan). The plasma levels of total adiponectin and leptin were measured using ELISAs (Otsuka Pharmaceutical, Tokyo, Japan, and Morinaga, respectively). The triacylglycerol content of the liver was determined as described elsewhere [10]. HOMA of insulin resistance (HOMA-IR) was calculated by using the formula [fasting insulin $(\mathrm{mU} / \mathrm{l}) \times$ fasting plasma glucose $(\mathrm{mmol} / \mathrm{l})] / 22.5$. When insulin is expressed in SI units as $\mathrm{pmol} / \mathrm{l}$, the constant changes to 156.26 .

Glucose tolerance test Mice were denied access to food for more than $16 \mathrm{~h}$ before the study and then orally loaded with glucose at $1.5 \mathrm{mg} / \mathrm{g}$ body weight. Blood samples were collected before, and at 15, 30, 60 and 120 min after glucose loading.

Insulin tolerance test The insulin tolerance test was performed under non-fasting conditions. Insulin was injected intraperitoneally, and blood samples were collected before, and at 30, 60, 90 and $120 \mathrm{~min}$ after the injection.

Histopathological evaluation Liver samples were immersionfixed overnight in $10 \%$ formalin (vol./vol.) at $4^{\circ} \mathrm{C}$. The tissues were then routinely processed for paraffin embedding, and $5 \mu \mathrm{m}$ sections mounted on glass slides were processed for haematoxylin and eosin (H\&E) staining. The presence of collagen, which can be used as an index of fibrosis in lesions, was examined using Masson trichromestained preparations.

Liver histology and scoring system All the histopathological findings were scored by experienced pathologists, who were unaware of the genetic backgrounds and diets of the mice. The histological features were grouped into three broad categories: steatosis, inflammation and fibrosis. The scoring system used for the evaluation is detailed in ESM Table 2.

\section{$R N A$ preparation and real-time quantitative $P C R$ Total} RNA was prepared from portions of the liver using a reagent (Isogen; NipponGene, Tokyo, Japan), according to the manufacturer's instructions, and these samples were used as the starting material for cDNA preparations. cDNA was synthesised using reagents (TaqMan Reverse Transcription; Applied Biosystems, Foster City, CA, USA), followed by TaqMan quantitative PCR $\left(50^{\circ} \mathrm{C}\right.$ for $2 \mathrm{~min}$ and $95^{\circ} \mathrm{C}$ for $10 \mathrm{~min}$, followed by 40 cycles at $95^{\circ} \mathrm{C}$ for $15 \mathrm{~s}$ and at $60^{\circ} \mathrm{C}$ for $1 \mathrm{~min}$ ), performed on a PCR instrument (ABI Prism 7500; Applied Biosystems) to amplify the following genes: Pparg, Srebplc (also known as Srebf1), Fas, Scd1, Ppara, Cpt1 (also known as Cpt1a), Mcad (also known as Acadm), Tnfa (also known as Tnf), Mcp1 (also known as Ccl2), p22phox (also known as $C y b a$ ), gp91phox (also known as Cybb), p47phox (also known as $N c f 1$ ) and $I r s 2$. The relative expression levels were then compared after normalisation to the expression of beta-actin.

Statistical analysis Results are expressed as means $\pm \operatorname{SEM}(n)$. Differences between two groups were analysed for statistical significance using Student's $t$ test. Individual comparisons among four groups were performed using an ANOVA followed by Fisher's protected least significant difference post-hoc test. Also, individual comparisons between two time points and two groups were performed using a two-way ANOVA. A value of $p<0.05$ was considered statistically significant.

\section{Results}

Effects of long-term HF diet on metabolic changes in C57Bl/6J mice First, we tested the effect of long-term HF diet loading on the development of NASH and liver tumourigenesis in $\mathrm{C} 57 \mathrm{Bl} / 6 \mathrm{~J}$ male (wild-type) mice. After 30 and 60 weeks on the HF diet, this mouse model showed higher body weights, fasting blood glucose levels, liver weights and plasma ALT levels than mice fed SC, but the plasma ALT levels were not significantly higher, presumably because of an interaction effect (Fig. 1a-d). The triacylglycerol content of the liver tended to be higher in wildtype mice after 30 , but not after 60 weeks on the HF diet, compared with that in mice fed the SC diet (Fig. 1e). The glucose-lowering effect of insulin was impaired in this mouse model when fed the HF diet, compared with that in the same mouse model on the SC $\operatorname{diet}$ (Fig. 1f). Thus, the long-term administration of an HF diet induced obesity and insulin resistance in $\mathrm{C} 57 \mathrm{Bl} / 6 \mathrm{~J}$ male mice. Furthermore, fasting insulin levels, HOMA of insulin resistance (HOMA-IR) and leptin levels were significantly higher, while plasma adiponectin levels were significantly lower in animals fed the HF diet than in those fed the SC diet (Fig. 1g-j), consistent with the results of previous reports $[17,18]$.

Effects of long-term HF diet on the risk of occurrence of NASH and liver tumourigenesis in C57Bl/6J mice Livers from wild-type mice fed the HF diet for 60 weeks were enlarged, compared with those in animals fed the SC diet for the same duration (Fig. 2a). Whereas wild-type mice on the SC diet had an almost normal liver histology (Fig. 2b, e), those fed the HF diet had typical features of NASH (Fig. 2c) at various stages of progression in the liver, including portal inflammation (Fig. 2d) and blue wave-like bands of fibrotic tissue in portal lesions (Fig. 2f, g). Scoring of the pathological findings showed significant increases for liver steatosis, inflammation and fibrosis in wild-type mice fed the HF diet, compared with scores in their counterparts on the SC diet (Fig. 2h). The expression of lipogenic genes, such as Fas and $S c d 1$, after 30 weeks (Fig. 2i), and of genes encoding 
a

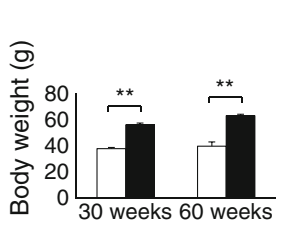

C
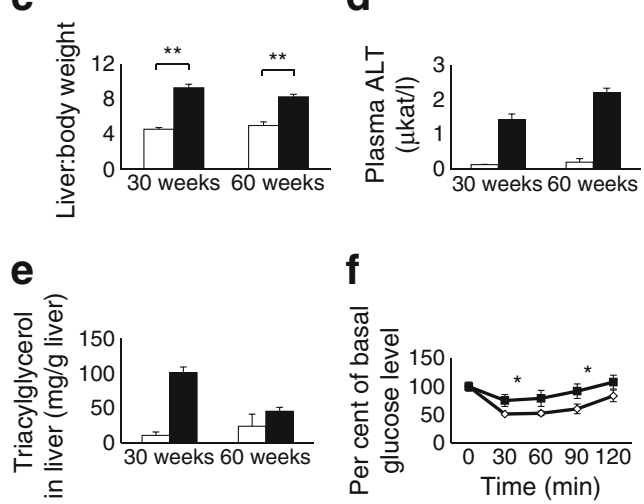

b

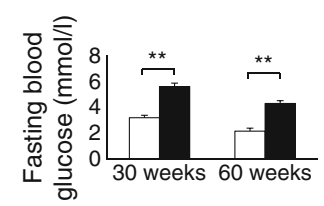

d
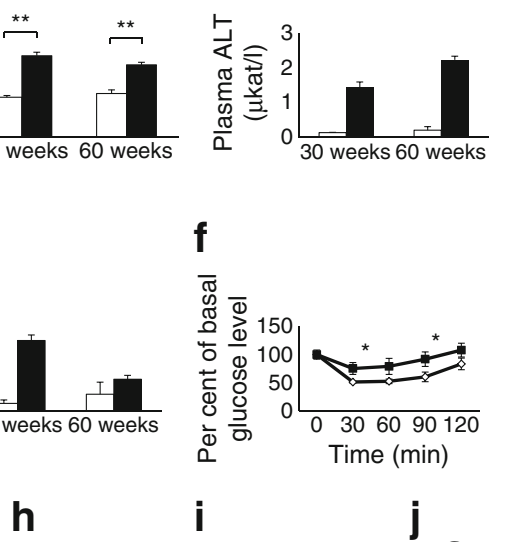

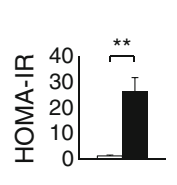

i

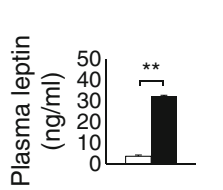

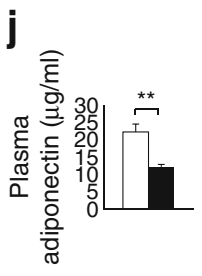

Fig. 1 Effects of a long-term HF diet on metabolic changes in C57B1/ $6 \mathrm{~J}$ mice. (a) Body weight, (b) fasting blood glucose, (c) ratio of liver: body weight, (d) plasma ALT and (e) triacylglycerol content of liver in mice fed the SC (white bars) or HF (black bars) diet for 30 or 60 weeks $(n=8-24)$. (f) Insulin tolerance test in mice fed the SC (white diamonds) or HF (black squares) diet for 60 weeks $(n=6)$. (g) Plasma fasting insulin levels, (h) HOMA-IR calculated from fasting blood glucose and insulin levels, (i) leptin levels and (j) total adiponectin levels in mice fed the SC or HF diet for 30 weeks $(n=10-11)$. Values are mean \pm SEM; $* p<0.05$ and $* * p<0.01$

inflammatory cytokines, such as Tnfa and $M c p l$, after 60 weeks (Fig. 2j) was significantly increased in wild-type mice fed the HF diet, compared with that in animals fed the $\mathrm{SC}$ diet. Furthermore, the expression of genes encoding the reduced-form NADPH oxidase complex was coordinately elevated after 60 weeks in mice fed the HF diet, compared with findings in animals fed the SC diet (Fig. 2k). These results reflect the natural course of NASH: in other words, healthy liver becomes steatotic, followed by an inflammatory process caused by cytokines and oxidative stress, which results in hepatocellular degeneration and fibrosis. Moreover, tumours of various diameters were frequently observed on the liver surface in the HF group (Fig. 21). Pathologically, these tumours were dysplastic nodules, adenomas or welldifferentiated carcinomas (Fig. $2 \mathrm{~m}, \mathrm{n}$ ), consistent with the findings of previous reports [6,7]. These nodular lesions were observed on the liver surface in $10 \%$ of animals after 30 weeks and in $54 \%$ of animals after 60 weeks of

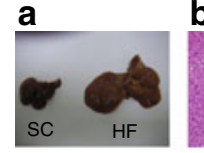

b

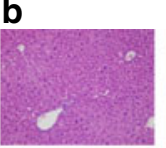

e

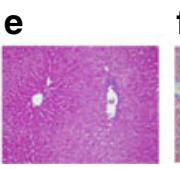

c

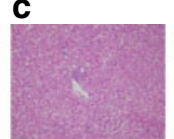

d

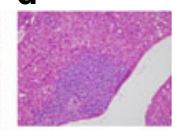

f

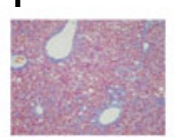

g

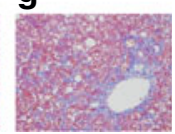

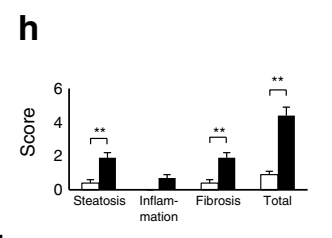

i
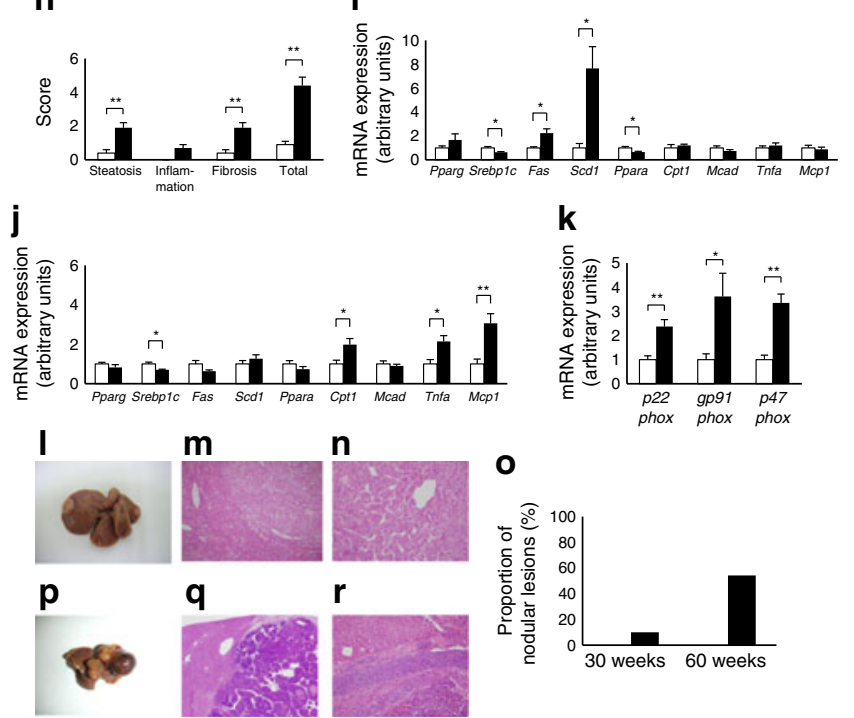

k

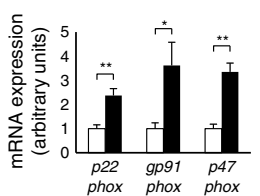

o

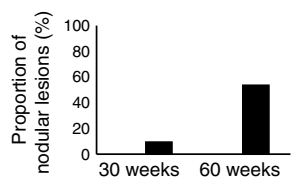

Fig. 2 Effects of a long-term HF diet on the risk of NASH and liver tumourigenesis in $\mathrm{C} 57 \mathrm{Bl} / 6 \mathrm{~J}$ mice. (a) Macroscopic findings after 60 weeks. (b, e) Histopathological findings in the livers of mice fed the SC or HF (c, d, f, g) diets, as assessed using H\&E- (b-d) and Masson trichrome (e-g)-stained sections. (h) The NASH/NAFLD Clinical Research Network scoring system definition and scores [32] for mice fed the SC (white bars) or HF (black bars) diet $(n=9)$. (i) mRNA expression of lipogenic and inflammatory cytokine-related genes as listed after 30 and $60(\mathbf{j})$ weeks, and (k) of genes encoding the reduced-form NADPH oxidase complex after 60 weeks in mice fed the SC or HF diet $(n=5-6)$. (I) Macroscopic and $(\mathbf{m}, \mathbf{n})$ histopathological findings in sections containing liver tumours, as assessed using H\&Estained sections from mice fed the HF diet for 60 weeks. (o) Proportion of hepatic nodular lesions in mice fed the SC or HF diet $(n=8-24)$. (p) Macroscopic and $(\mathbf{q}, \mathbf{r})$ histopathological features as assessed using H\&Estained sections of hepatic tumours from mice fed the HF diet for 80 weeks. Values are mean $\pm \mathrm{SEM} ;{ }^{*} p<0.05$ and ${ }^{* *} p<0.01$

administration of the HF diet in wild-type mice. In contrast, no such nodular lesions were detected in mice fed the SC diet (Fig. 20). Interestingly, HCCs, which are characterised by disrupted normal liver architecture and intravascular tumour embolism, were found in certain mice after 80 weeks on the HF diet (Fig. 2p-r). As a point of reference, no increase in the phosphorylation levels of c-Jun N-terminal kinase (JNK), extracellular signal-regulated kinase (ERK) or $\mathrm{p} 38$ were noted in wild-type mice fed the HF diet, compared with the levels in mice fed the SC diet (data not shown). These results indicate that the long-term HF diet loading was sufficient to induce NASH and liver tumourigenesis in wild-type mice. 
Switching from the HF to the SC diet: effects on metabolic changes in C57Bl/6J mice Next, to evaluate the effects of nutrients on fat distribution within the body, the degree of insulin resistance, and the risk of NASH and liver tumourigenesis, we compared $\mathrm{C} 57 \mathrm{Bl} / 6 \mathrm{~J}$ male mice that were fed the HF diet for 30 weeks, followed by 30 weeks on the SC diet, with mice from the same genetic background that were fed the HF diet for the entire 60 weeks. The mice that were switched from the HF diet to the SC diet showed a significantly lower body weight and visceral fat weight than the animals that were fed the HF diet for the entire 60 weeks, although no differences in fed-state blood glucose levels were observed between the two groups (Fig. 3a-d). The diet switch improved the degree of hyperinsulinaemia and insulin resistance, as evidenced by fasting insulin levels, HOMA-IR and insulin tolerance test results (Fig. 3e, f, ESM Fig. 1). The diet switch was also associated with a decrease in liver weight, plasma ALT levels and liver triacylglycerol content, although the difference for the latter was not statistically significant (Fig. $3 g-j$ ).

Switching from the HF to the SC diet: effects on the incidence of NASH and liver tumourigenesis in C57Bl/6J mice Compared with the findings for mice that continued the HF diet for the entire 60 weeks, findings for mice that were switched from the HF to the SC diet showed an almost normal liver histology (Fig. 4a, b), with significantly decreased pathological scores for NASH (Fig. 4c). Also, the expression of genes encoding inflammatory cytokines such as Tnfa and $M c p l$, and the reducedform NADPH oxidase complex decreased significantly in the group that switched diets (Fig. 4d, e). The proportion of nodular lesions was also significantly decreased by the dietary switch (Fig. 4f). These findings suggest that the correction of nutrient conditions improved obesity and the related insulin resistance, and protected the animals against HF diet-induced NASH and liver tumourigenesis.

Effects of long-term HF diet on metabolic changes in Irs $1^{--}$ mice To evaluate the effect of hepatic steatosis on HF dietinduced NASH and liver tumourigenesis, we used $\operatorname{Irs} 1^{-/}$ male mice, which exhibit postnatal growth retardation and insulin resistance, but have normal glucose tolerance because of compensatory beta cell hyperplasia [19-21]. As expected, the body weight of $\operatorname{Irs} 1^{-/-}$mice was about two thirds that of wild-type mice in the SC and HF diet groups (Fig. 5a). However, fed-state blood glucose levels were increased in $\mathrm{Irs} \mathrm{I}^{-/-}$ mice, compared with those in wild-type mice, after 6 weeks on the HF diet, although no differences in fed-state glucose levels were observed between wild-type and $I r s 1^{-/}$mice fed the SC diet (Fig. 5b). Interestingly, the liver weight, plasma ALT levels and triacylglycerol content of the liver were significantly higher in wild-type mice fed the HF diet than in the other groups of mice (Fig. 5c-e). Moreover, although wild-type
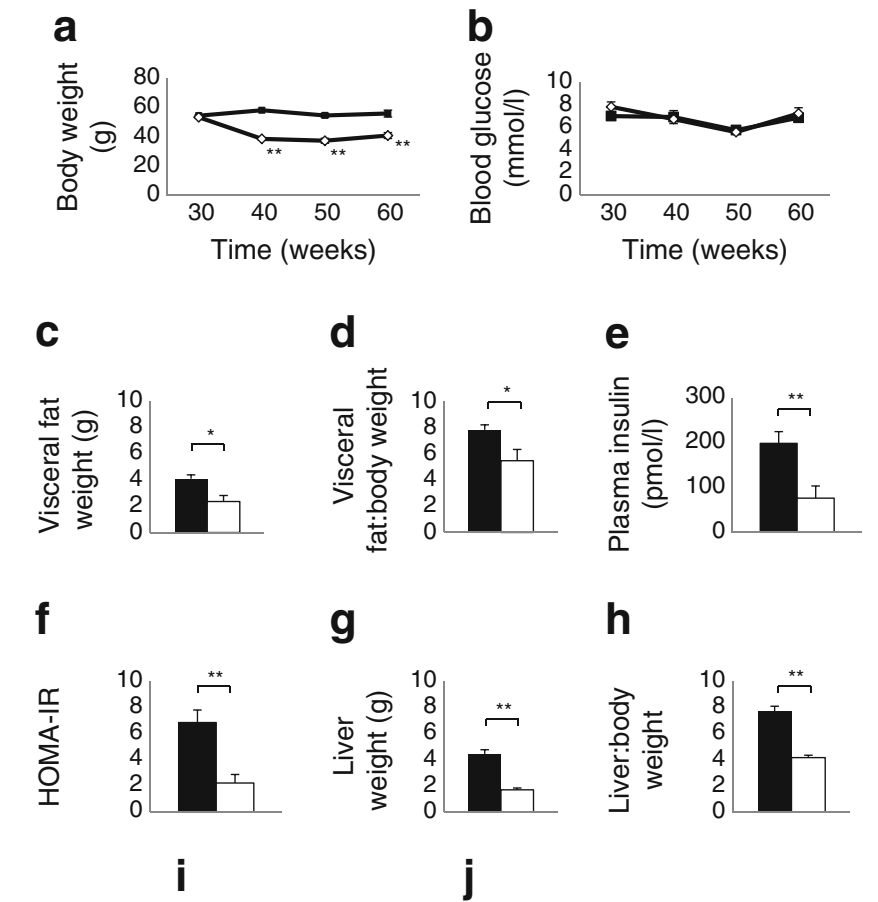

\section{g}

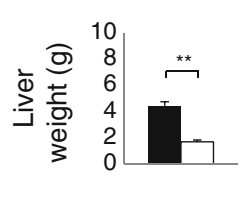

h
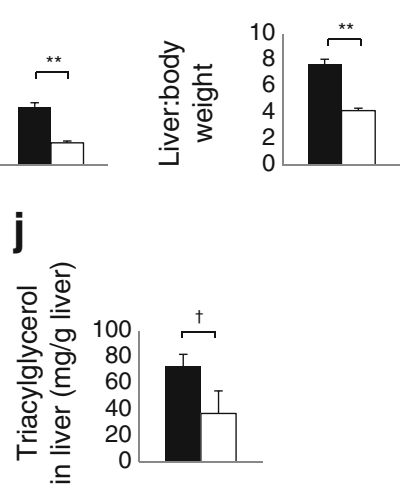

Fig. 3 Effects of the switch from the HF to the SC diet on metabolic changes in $\mathrm{C} 57 \mathrm{Bl} / 6 \mathrm{~J}$ mice. The mice were fed the HF diet for 30 weeks, followed by the SC diet for 30 weeks. They were then compared with mice fed the HF diet for 60 weeks. (a) Changes in body weight and (b) fed-state blood glucose levels in the HF diet alone (black squares) and $\mathrm{HF}+\mathrm{SC}$ diet (white diamonds) groups $(n=10)$. (c) Visceral fat weight and (d) the ratio of visceral fat weight to body weight in the HF diet alone (black bars) and the HF $+\mathrm{SC}$ diet (white bars) groups $(n=5)$. (e) Plasma fasting insulin, (f) HOMA-IR, (g) liver weight, (h) ratio of liver to body weight, (i) plasma ALT and (j) triacylglycerol content of the liver in the two groups of mice $(n=10)$. Values are mean \pm SEM; ${ }^{*} p<$ 0.05 and $* * p<0.01 ;{ }^{\dagger} p=0.08$

mice fed the HF diet demonstrated the typical features of hepatic steatosis, the other three groups showed an almost normal liver histology (Fig. 5f-i). These results indicate that the disruption of IRS-1 protected against HF diet-induced hepatic steatosis.

Based on these results, we next compared the effects of long-term HF diet loading on metabolic changes in $\mathrm{Irs} \mathrm{I}^{-/-}$ mice relative to those in wild-type mice. Although the body weight of $\operatorname{Irs} 1^{-/-}$mice was about two thirds that of wildtype mice and the visceral fat weight in the former was also lower ( $\operatorname{Irs} 1^{-/}$mice $2.3 \pm 0.1 \mathrm{~g}$ vs wild-type $3.9 \pm 0.1 \mathrm{~g}$ ), no difference in the ratio of visceral weight to body weight was observed between the two groups (Fig. 6a-c). The insulin and oral glucose tolerance tests revealed that $\operatorname{Irs} 1^{-/-}$mice 
a

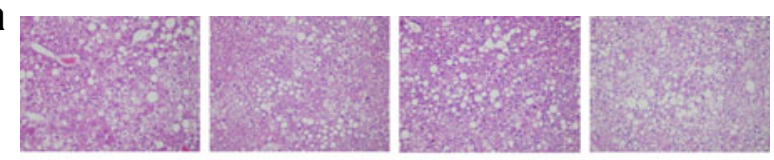

b

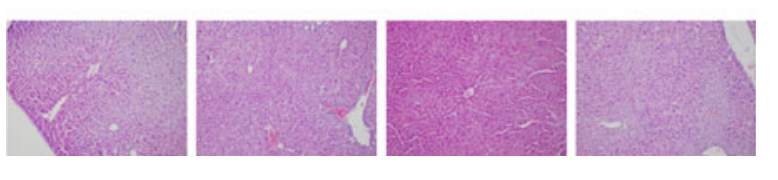

C

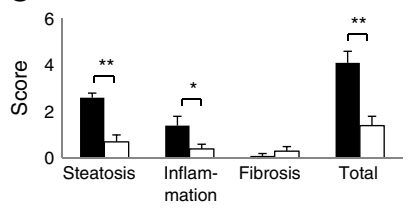

d
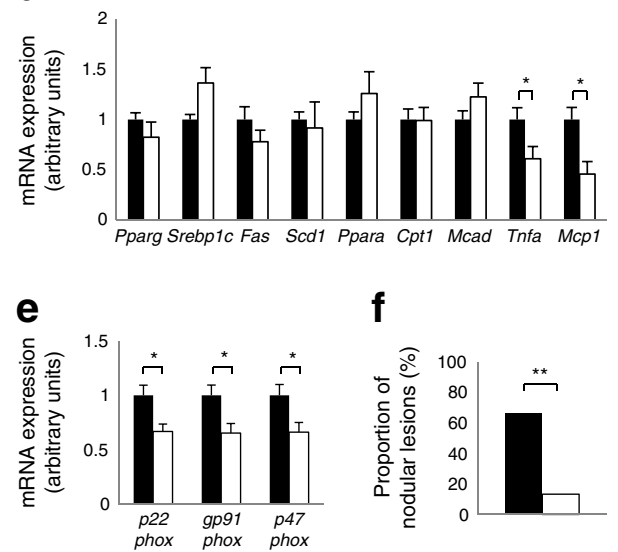

Fig. 4 Effects of switching from the HF to the SC diet on the incidence of NASH and liver tumourigenesis in $\mathrm{C} 57 \mathrm{Bl} / 6 \mathrm{~J}$ mice. The mice were fed the HF diet for 30 weeks, followed by the SC diet for 30 weeks. They were then compared with mice fed the HF diet for 60 weeks. (a) Histopathological findings of NASH in the livers from the HF diet alone group and (b) the HF + SC diet group, as observed using H\&E-stained sections $(n=4)$. (c) NASH/NAFLD Clinical Research Network scoring system definitions and scores [32] for the HF diet alone (black bars) and the HF $+\mathrm{SC}$ diet (white bars) groups of mice $(n=9-10)$. (d) mRNA expression of lipogenic and inflammatory cytokine-related genes, and (e) of genes encoding the reduced-form NADPH oxidase complex in the two groups of mice $(n=4-6)$. (f) Proportion of hepatic nodular lesions in the two groups of mice $(n=$ $15)$. Values are mean $\pm \mathrm{SEM} ;{ }^{*} p<0.05$ and ${ }^{* *} p<0.01$

fed the HF diet had severe insulin resistance and marked postprandial hyperglycaemia compared with wild-type mice on the same diet (Fig. 6d, e). Also, their fasting insulin levels and HOMA-IR values were significantly higher than in wild-type mice (Fig. 6f, g). Moreover, the liver weight, plasma ALT levels and triacylglycerol content of the liver were significantly lower in $\operatorname{Irs} 1^{-/-}$mice than in wild-type mice fed the HF diet (Fig. 6a, h-j). Although no difference in plasma leptin levels was seen, adiponectin levels were significantly higher in $\operatorname{Irs} 1^{-/}$ mice (Fig. 6k, 1). a
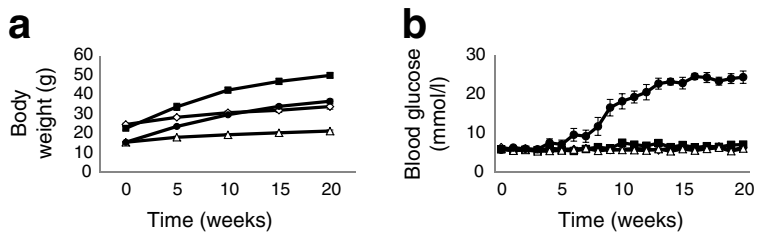

C

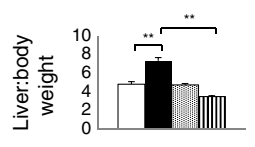

d
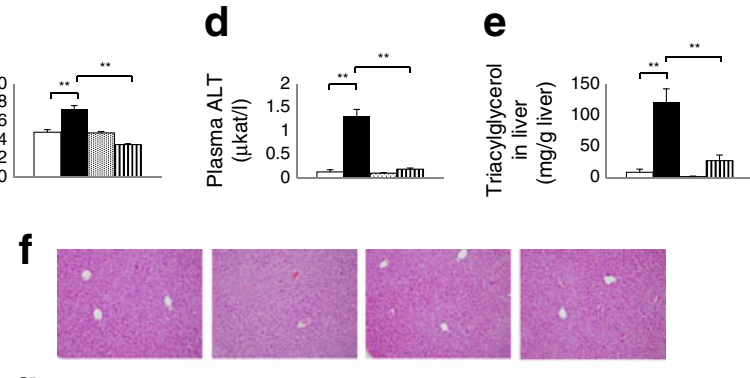

g
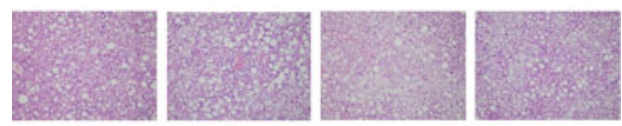

h
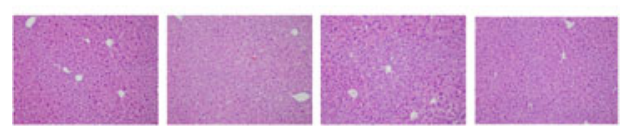

i
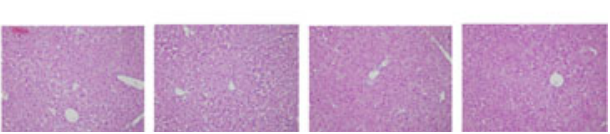

Fig. 5 Effects of an HF diet on liver steatosis in $\operatorname{Irs} 1^{-/}$mice. (a) Changes in the body weight and (b) fed-state blood glucose levels in wild-type and $\mathrm{Irs} \mathrm{I}^{-/-}$mice fed the SC or HF diet (white diamonds, wild-type SC; black squares, wild-type HF; white triangles, $\mathrm{Irs} 1^{-/-} \mathrm{SC}$; black circles, Irs $1^{-/}$HF) $(n=7-11)$. (c) Ratio of liver weight to body weight, (d) plasma ALT and (e) triacylglycerol content of the liver in mice as above $(\mathbf{a}, \mathbf{b})$ at 20 weeks of respective diet (white bars, wildtype SC; black bars, wild-type HF; dotted bars, Irs $1^{-/} \mathrm{SC}$; striped bars, Irs $\left.1^{-/-} \mathrm{HF}\right)(n=5-10)$. (f) Histopathological findings in the livers of wild-type SC-, (g) wild-type HF-, (h) $I r s 1^{-/} \mathrm{SC}$ - and (i) $\mathrm{Irs} 1^{-/-} \mathrm{HF}$ fed mice, as assessed using H\&E-stained sections $(n=4)$. Values are mean \pm SEM; $* * p<0.01$

Effects of long-term HF diet on the incidence of NASH and liver tumourigenesis in Irs $1^{-/}$mice Apparently, Irs $1^{-/-}$ mice fed the HF diet showed an almost normal liver histology (Fig. 7a-d), while pathological scores for NASH were significantly lower in $\operatorname{Irs} 1^{-/}$mice (Fig. 7e). Also, the expression of lipogenic genes such as Fas and Scdl at 30 weeks, and of genes encoding inflammatory cytokines and the reduced-form NADPH oxidase complex at 60 weeks were significantly decreased in $I r s 1^{-/}$mice on the HF diet, compared with wild-type mice fed the same diet (Fig. $7 \mathrm{f}-\mathrm{h}$ ). Moreover, the proportion of nodular lesions was significantly lower in $\operatorname{Irs} 1^{--}$mice fed the HF diet than in wild-type mice on the same diet (Fig. 7i). These results indicate that the disruption of IRS-1 protected against HF diet-induced NASH and liver tumourigenesis, despite being associated with severe hyperglycaemia and insulin resistance. 
a
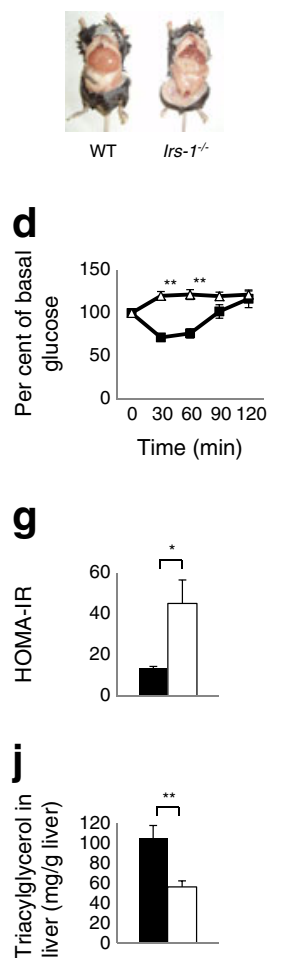

b

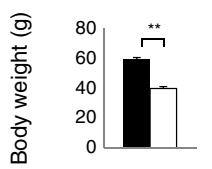

e

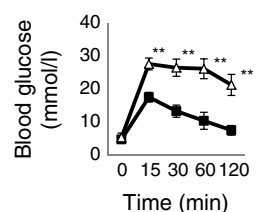

h

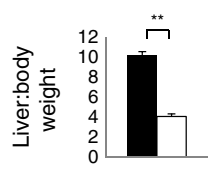

$\mathbf{k}$

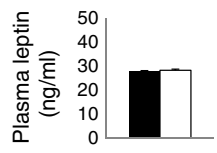

C

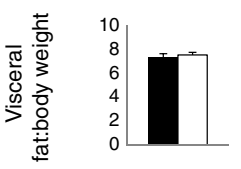

f

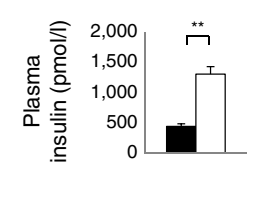

i

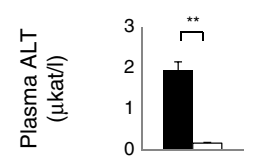

I

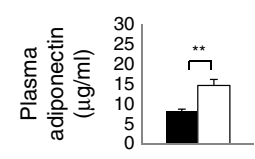

Fig. 6 Effects of a long-term HF diet on metabolic changes in Irs $1^{-1-}$ mice. (a) Macroscopic observation in wild-type (WT) and $\mathrm{Irs} 1^{-/-}$mice fed the HF diet. (b) Body weight and (c) ratio of visceral weight to body weight in wild-type (black bars) and $I r s 1^{-/-}$(white bars) mice fed the HF diet for 30 weeks $(n=4-10)$. (d) Insulin and (e) oral glucose tolerance tests in wild-type (black squares) and $\operatorname{Irs} 1^{-1-}$ (white triangles) mice fed the HF diet for 30 weeks $(n=8-10)$. (f) Plasma fasting insulin, (g) HOMA-IR, (h) ratio of liver weight to body weight, (i) plasma ALT, (j) hepatic triacylglycerol content, (k) plasma leptin levels and (I) plasma total adiponectin levels in wild-type and $I r s 1^{-/}$mice fed the HF diet for 30 weeks $(n=6-10)$. Values are mean $\pm \mathrm{SEM}$; ${ }^{*} p<0.05$ and $* * p<0.01$

\section{Discussion}

In the present study, we showed that long-term HF diet loading, which causes obesity and peripheral insulin resistance, was sufficient to induce NASH and liver tumourigenesis in $\mathrm{C} 57 \mathrm{Bl} / 6 \mathrm{~J}$ mice, and that the reduction of obesity and peripheral insulin resistance by switching from the HF to an SC diet protected animals against the development of NASH and liver tumourigenesis. More importantly, the results of our study indicate that $\mathrm{Irs} \mathrm{I}^{-/-}$mice fed the HF diet were dramatically protected against NASH and liver tumourigenesis, despite having severe insulin resistance and marked postprandial hyperglycaemia. These results are not consistent with the prevalent notion that the insulin resistance associated with obesity and diabetes is involved in the development of hepatic steatosis and inflammation in the liver, which may progress to NASH and liver tumourigenesis [5].

How can these results be explained? One explanation is the concept of selective or partial insulin resistance [22]. Thus humans with insulin resistance caused by inherited mutations in the insulin receptor and mice with a liver-specific deletion of the insulin receptor have hyperglycaemia and hyperinsulinaemia, but are both protected against hepatic steatosis and hypertriacylglycerolaemia [23, 24]. This finding is consistent with the idea that not all signals are blunted in classical insulin-resistant states; instead, some signals are preserved, particularly those related to hepatic steatosis.

Insulin receptor signalling can be almost exclusively mediated by IRS- 1 and IRS- 2 in the liver, with IRS-2 mainly functioning during fasting and immediately after re-feeding, while IRS-1 functions primarily after re-feeding [10]. In our results in wild-type mice, Irs 2 levels were significantly decreased in the HF diet group, compared with those in the SC diet group, under fasting conditions, while levels of Irs 1 in the HF diet group were similar to those in the SC diet group under re-feeding conditions (ESM Fig. 2). Insulin signalling might be decreased mainly under fasting conditions in the HF diet group, as the hyperinsulinaemia associated with an HF diet may suppress IRS-2 production [25]. In this case, HF diet feeding might place the mice in a chronic postprandial state that preferentially inactivates IRS-2, with persistent IRS-1 signalling possibly promoting lipogenesis and leading to hepatic steatosis, since IRS-1 has been proposed to be the dominant regulator of expression of the hepatic genes controlling lipogenesis [11]. In contrast, hepatic insulin signalling in $I r s 1^{-/-}$mice fed the HF diet was impaired, since IRS- 1 was absent and IRS-2 signalling was suppressed by the hyperinsulinaemia associated with the HF diet. Thus, the pathophysiological features in $\operatorname{Irs} 1^{-/}$mice fed the HF diet might be similar to those in liver-specific Irs 1/ Irs 2 double-knockout mice and in liver-specific insulin receptor knockout mice [11, 24]. A similar situation is seen with the liver-specific loss-of-function of the p110 $\alpha$ subunit of PI3K [13] or of Akt [26]. Since the IRS proteins lie between these steps [27], these previous studies using mouse models with genetic engineering of genes encoding the insulin receptor, IRS, PI3K and Akt are consistent with the phenotype of the $\operatorname{Irs} 1^{-1}$ mice fed the HF diet in our study.

Importantly, the mice in the present study, unlike liverspecific knockout mice, had impaired IRS-1 functions in all their tissues. It thus remains unclear whether the protection against NASH and liver tumourigenesis is due to a global loss of insulin signalling or a liver-specific loss. Unfortunately, the current data do not answer this question. However, we assumed that the protection might be due to a liverautonomous effect, since liver-specific $I r s 1^{-/}$mice fed an HF diet, but not liver-specific Irs $2^{-/-}$mice fed an HF diet were reportedly protected from hepatic steatosis [11] and the steatotic host microenvironment probably sets the stage for tumour development, even during the initially reversible and treatable stages of fatty liver disease [7]. Therefore, this 
Fig. 7 Effects of a long-term HF diet on the incidence of $\mathrm{NASH}$ and liver tumourigenesis in $I r s 1^{-/}$mice. (a) Histopathological features of the livers from wild-type and (b) $I r s 1^{-/-}$ mice fed the HF diet for 60 weeks, as assessed using H\&E-stained sections $(n=4)$.

(c) Histopathological features of the livers from wild-type and (d) $I r s 1^{-/-}$mice fed the HF diet for 60 weeks, as assessed using Masson trichrome-stained sections $(n=4)$. (e) NASH/NAFLD Clinical Research Network scoring system definitions and scores [32] for wild-type (black bars) and $I r s 1^{-1-}$ (white bars) mice fed the HF diet for 60 weeks $(n=9)$. (f) mRNA expression of lipogenic and inflammatory cytokine-related genes after 30 and (g) 60 weeks on the HF diet, and (h) of genes encoding the reduced-form NADPH oxidase complex after 60 weeks on the HF diet in wild-type and $I r s 1^{-/}$mice $(n=4-6)$. (i) Proportion of hepatic nodular lesions in wildtype and $I r s 1^{-/-}$mice fed the HF diet for 60 weeks $(n=11)$.

Values are mean $\pm \mathrm{SEM}$; ${ }^{*} p<0.05$ and $* * p<0.01 ;{ }^{\dagger} p=$ 0.05
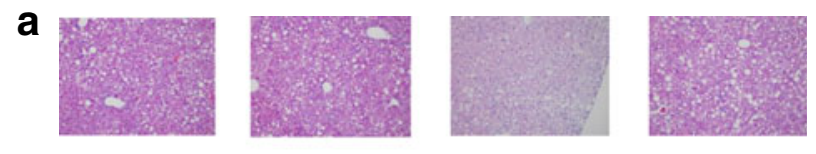

b
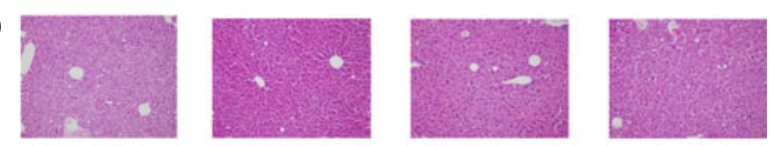

C
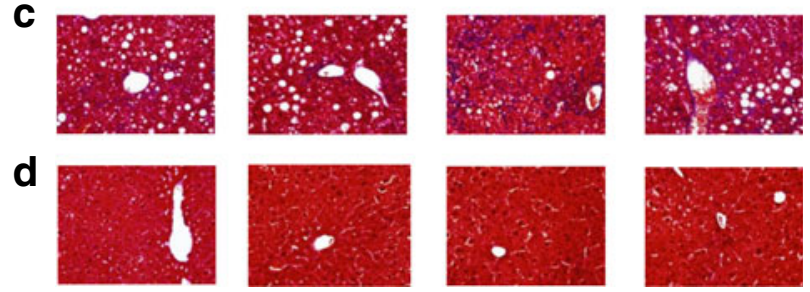

e f
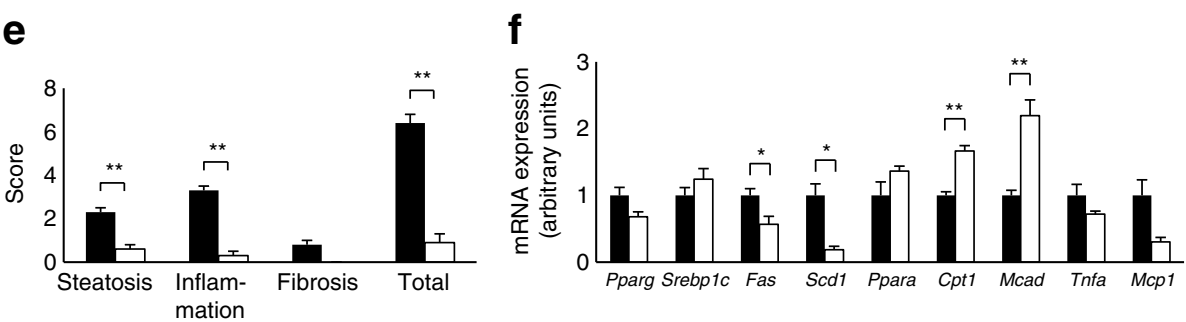
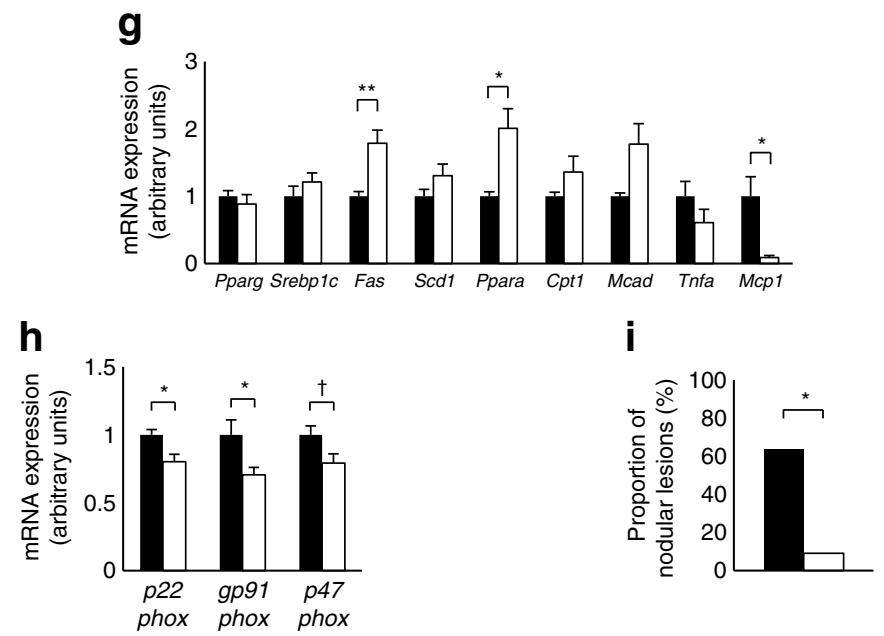

hypothesis should be further examined using liver-specific $\operatorname{Irs} 1^{-/-}$and $\operatorname{Irs} 2^{-/-}$mice [10] in the future.

Another question that remains unclear is whether the contribution or compensation of IRS-2 signalling plays a role in the above-mentioned protective effect. Our data show that $I r s 2$ expression in $\operatorname{Irs} 1^{-/}$mice on the HF diet was significantly higher than in wild-type mice fed the HF diet. However, levels were significantly lower than those in wild-type mice fed the SC diet. Moreover, the basal and insulin-stimulated phosphorylation of Akt in $\mathrm{Irs} \mathrm{I}^{-/-}$mice on the HF diet was lower than in wild-type mice fed the HF diet (data not shown). These results suggest that IRS-2 may not fully compensate for the loss of IRS-1 in $\operatorname{Irs} 1^{-/-}$mice on an HF diet. We therefore assumed that the contribution of residual IRS-2 signalling was limited to the livers of $I r s 1^{-/}$ mice on the HF diet.

What is the relevance of the present results to the clinical management of humans with diabetes? Therapeutic targeting of IRS-1 may not be advisable, since IRS1 is an insulin resistance gene in humans [28] and our results suggest that blocking IRS1-mediated signalling exacerbated glucose tolerance, even though it was able to protect against NASH and liver tumourigenesis. Epidemiological evidence suggests that people with diabetes have significantly higher risks of many forms of cancer [29]. Recently, a meta-analysis of several studies showed that liver cancer is more common in patients with diabetes [30]. 
Johnson et al commented that the accumulation of experimental and epidemiological evidence is more consistent with the hyperinsulinaemia hypothesis and less so with the hyperglycaemia hypothesis [31] with regard to the increased risk of cancer in patients with diabetes. Here, we showed that the reduction of obesity and hyperinsulinaemia by switching from an HF to an SC diet protected mice against the development of NASH and liver tumourigenesis without changing blood glucose levels. These results support the hyperinsulinaemia hypothesis. How can our results for $I r s 1^{-/-}$mice fed an HF diet be explained? We assumed that the protection against NASH and tumour development in $I r s 1^{-/-}$mice fed the HF diet was caused by the downregulation of IRS-1-mediated insulin action in the liver, despite systemic hyperinsulinaemia. As described above, the hyperinsulinaemia associated with an HF diet suppresses IRS2 production, and persistent IRS-1 signalling promotes lipogenesis and hepatic steatosis. Thus, when mice were fed the HF diet, the wild-type mice developed hepatic steatosis, but the $I r s 1^{-/}$ mice were protected against the development of NASH and liver tumourigenesis, despite the presence of hyperinsulinaemia. Thus, these results for $I r s 1^{--}$mice fed an HF diet are consistent with the above-mentioned hyperinsulinaemia hypothesis. Therefore, the prevention of hyperinsulinaemia using glucoselowering agents such as metformin and thiazolidinedione could not only protect against diabetes, but also against liver tumourigenesis. The effects of metformin on NASH and liver tumourigenesis in this mouse model are now under investigation.

In conclusion, long-term HF diet loading was sufficient to induce NASH and liver tumourigenesis in $\mathrm{C} 57 \mathrm{Bl} / 6 \mathrm{~J}$ mice. Switching from an HF to an SC diet reduced obesity and insulin resistance, and protected against the development of NASH and liver tumourigenesis in the same mice. Moreover, $\operatorname{Irs} \mathrm{I}^{-/}$mice fed an HF diet were dramatically protected against NASH and liver tumourigenesis, suggesting that IRS-1 inhibition might protect against HF diet-induced NASH and liver tumourigenesis, despite the presence of insulin resistance.

Acknowledgements We thank M. Kaji and E. Sakamoto (Department of Endocrinology and Metabolism, Graduate School of Medicine, Yokohama City University, Yokohama, Japan) for their excellent technical assistance and animal care.

Funding This work was supported in part by: Grants-in-Aid for Scientific Research (B) 19390251 and (B) 21390282 from the Ministry of Education, Culture, Sports, Science and Technology (MEXT) of Japan; a Medical Award from the Japan Medical Association; a Grantin-Aid from the Uehara Memorial Foundation; a Grant-in-Aid from the Daiichi-Sankyo Foundation of Life Science; and a Grant-in-Aid from the Naito Foundation (to Y. Terauchi).

Contribution statement All the authors conceived and designed the study, and participated in the analysis and interpretation of the data. AN drafted the manuscript, and all the other authors revised it critically for intellectual content. All the authors approved the final version of the paper.
Duality of interest The authors declare that there is no duality of interest associated with this manuscript.

\section{References}

1. Finucane MM, Stevens GA, Cowan MJ et al (2011) National, regional, and global trends in body-mass index since 1980: systematic analysis of health examination surveys and epidemiological studies with 960 country-years and 9.1 million participants. Lancet 377:557-567

2. Danaei G, Finucane MM, Lu Y et al (2011) National, regional, and global trends in fasting plasma glucose and diabetes prevalence since 1980: systematic analysis of health examination surveys and epidemiological studies with 370 country-years and 2.7 million participants. Lancet 378:31-40

3. Calle EE, Rodriguez C, Walker-Thurmond K, Thun MJ (2003) Overweight, obesity, and mortality from cancer in a prospectively studied cohort of U.S. adults. N Engl J Med 348:1625-1638

4. Inoue M, Iwasaki M, Otani T, Sasazuki S, Noda M, Tsugane S (2006) Diabetes mellitus and the risk of cancer: results from a large-scale population-based cohort study in Japan. Arch Intern Med 166:1871-1877

5. Starley BQ, Calcagno CJ, Harrison SA (2010) Nonalcoholic fatty liver disease and hepatocellular carcinoma: a weighty connection. Hepatology 51:1820-1832

6. Hill-Baskin AE, Markiewski MM, Buchner DA et al (2009) Dietinduced hepatocellular carcinoma in genetically predisposed mice. Hum Mol Genet 18:2975-2988

7. VanSaun MN, Lee IK, Washington MK, Matrisian L, Gorden DL (2009) High fat diet induced hepatic steatosis establishes a permissive microenvironment for colorectal metastases and promotes primary dysplasia in a murine model. Am J Pathol 175:355-364

8. Park EJ, Lee JH, Yu GY et al (2010) Dietary and genetic obesity promote liver inflammation and tumorigenesis by enhancing IL-6 and TNF expression. Cell 140:197-208

9. Saltiel AR, Kahn CR (2001) Insulin signalling and the regulation of glucose and lipid metabolism. Nature 414:799-806

10. Kubota N, Kubota T, Itoh S et al (2008) Dynamic functional relay between insulin receptor substrate 1 and 2 in hepatic insulin signaling during fasting and feeding. Cell Metab 8:49-64

11. Guo S, Copps KD, Dong X et al (2009) The Irs1 branch of the insulin signaling cascade plays a dominant role in hepatic nutrient homeostasis. Mol Cell Biol 29:5070-5083

12. Monetti M, Levin MC, Watt MJ et al (2007) Dissociation of hepatic steatosis and insulin resistance in mice overexpressing DGAT in the liver. Cell Metab 6:69-78

13. Chattopadhyay M, Selinger ES, Ballou LM, Lin RZ (2011) Ablation of PI3K p110- $\alpha$ prevents high-fat diet-induced liver steatosis. Diabetes 60:1483-1492

14. Horie Y, Suzuki A, Kataoka E et al (2004) Hepatocyte-specific Pten deficiency results in steatohepatitis and hepatocellular carcinomas. J Clin Invest 113:1774-1783

15. Kudo Y, Tanaka Y, Tateishi K et al (2011) Altered composition of fatty acids exacerbates hepatotumorigenesis during activation of the phosphatidylinositol 3-kinase pathway. J Hepatol 55:14001408

16. Kubota N, Tobe K, Terauchi Y et al (2000) Disruption of insulin receptor substrate- 2 causes type 2 diabetes due to liver insulin resistance and lack of compensatory beta-cell hyperplasia. Diabetes 49:1880-1889

17. Terauchi Y, Matsui J, Kamon J et al (2004) Increased serum leptin protects from adiposity despite the increased glucose uptake in white adipose tissue in mice lacking p85alpha phosphoinositide 3-kinase. Diabetes 53:2261-2270 
18. Kubota N, Yano W, Kubota T et al (2007) Adiponectin stimulates AMP-activated protein kinase in the hypothalamus and increases food intake. Cell Metab 6:55-68

19. Tamemoto H, Kadowaki T, Tobe K et al (1994) Insulin resistance and growth retardation in mice lacking insulin receptor substrate-1. Nature 372:182-186

20. Araki E, Lipes MA, Patti ME et al (1994) Alternative pathway of insulin signalling in mice with targeted disruption of the IRS-1 gene. Nature 372:186-190

21. Terauchi Y, Iwamoto K, Tamemoto H et al (1997) Development of non-insulin-dependent diabetes mellitus in the double knockout mice with disruption of insulin receptor substrate-1 and beta cell glucokinase genes. Genetic reconstitution of diabetes as a polygenic disease. J Clin Invest 99:861-866

22. Brown MS, Goldstein JL (2008) Selective versus total insulin resistance: a pathogenic paradox. Cell Metab 7:95-96

23. Semple RK, Sleigh A, Murgatroyd PR et al (2009) Postreceptor insulin resistance contributes to human dyslipidemia and hepatic steatosis. J Clin Invest 119:315-322

24. Biddinger SB, Hernandez-Ono A, Rask-Madsen C et al (2008) Hepatic insulin resistance is sufficient to produce dyslipidemia and susceptibility to atherosclerosis. Cell Metab 7:125-134
25. Zhang J, Ou J, Bashmakov Y, Horton JD, Brown MS, Goldstein JL (2001) Insulin inhibits transcription of IRS-2 gene in rat liver through an insulin response element (IRE) that resembles IREs of other insulin-repressed genes. Proc Natl Acad Sci USA 98:3756-3761

26. Leavens KF, Easton RM, Shulman GI, Previs SF, Birnbaum MJ (2009) Akt2 is required for hepatic lipid accumulation in models of insulin resistance. Cell Metab 10:405-418

27. Kadowaki T, Ueki K, Yamauchi T, Kubota N (2012) SnapShot: insulin signaling pathways Cell 148:624 (Abstract)

28. Rung J, Cauchi S, Albrechtsen A et al (2009) Genetic variant near IRS1 is associated with type 2 diabetes, insulin resistance and hyperinsulinemia. Nat Genet 41:1110-1115

29. Giovannucci E, Harlan DM, Archer MC et al (2010) Diabetes and cancer: a consensus report. Diabetes Care 33:1674-1685

30. Vigneri P, Frasca F, Sciacca L, Pandini G, Vigneri R (2009) Diabetes and cancer. Endocr Relat Cancer 16:1103-1123

31. Johnson JA, Pollak M (2010) Insulin, glucose and the increased risk of cancer in patients with type 2 diabetes. Diabetologia 53:2086-2088

32. Kleiner DE, Brunt EM, Van Natta M et al (2005) Design and validation of a histological scoring system for nonalcoholic fatty liver disease. Hepatology 41:1313-1321 